\title{
CME: Tropical medicine (138789): self-assessment questionnaire
}

\author{
Authors: Edited by Arosha Dissanayake and Tahseen A Chowdhury
}

\section{DOI: $10.7861 /$ clinmed.SAQ.22.1}

SAQs and answers are ONLINE for RCP fellows and collegiate members

Format

Candidates are asked to choose the best answer from the five possible answers. This best of five format is used in many medical examinations; however, the questions are not intended to be representative of those used in the MRCP(UK) Part 1 or Part 2 Written Examinations.

The answering process

1 Go to https://cme.rcplondon.ac.uk

2 Log on using your usual RCP username and password

3 Select the relevant CME question paper

4 Answer all 10 questions by selecting the best answer from the options provided

5 Once you have answered all the questions, click on Submit

\section{Registering your external CPD credits}

Carrying out this activity allows you to claim two external CPD credits. These will be automatically transferred to your CPD diary, where you can review the activity and claim your points.

1. A45-year-old man was being treated for suspected leptospirosis and acute kidney injury. His blood pressure was $110 / 70 \mathrm{mmHg}$, heart rate $90 / \mathrm{min}$, respiratory rate $18 / \mathrm{min}$. He was oliguric.

Which of the following is the most important immediate step in management?

(a) Doxycycline $100 \mathrm{mg}$ twice daily.

(b) Fluid challenge with observation of urine output.

(c) Intravenous methyl prednisolone pulse.

(d) Noradrenaline infusion.

(e) Plasmapheresis.

2. A 65 -year-old man was admitted with a 10-day history of fever with chills, myalgia and haemoptysis. He was noted to be jaundiced and hypoxaemic (SpO2 92\% on room air). He had bilateral coarse crackles in his lungs. Blood pressure was $120 / 80 \mathrm{mmHg}$ and his urine output was $400 \mathrm{ml}$ over the previous 5 hours. His history included having gone white-water rafting 3 weeks ago.

\section{What is the most appropriate next step in his management?}
(a) Dialysis.
(b) Intravenous methylprednisolone.
(c) Intravenous ceftriaxone.
(d) Intubation and ventilation.
(e) Plasmapharesis.

3. A 40-year-old man was admitted with a 5-day history of fever, myalgia, conjunctival suffusion and jaundice. Leptospirosis was suspected.
Which of the foll
the diagnosis?
(a) Leptospirosis IgM ELISA.
(b) Leptospirosis microscopic agglutination titre.
(c) Leptospirosis qPCR.
(d) Leptospirosis culture.
(e) Urine dark ground microscopy for leptospira.

4. A 34-year-old engineer had returned to the UK 7 days previously after a 2-week trip to Sri Lanka. He had visited Yala National Park, a wildlife park, in southern Sri Lanka 2 days prior to departure. He felt generally unwell with high fevers, headache and body aches. He malaria parasite screen was negative, and full blood count and urine investigations were normal. He was observed for 4 days with no improvement. He complained of tinnitus on day 6 . He had no rash. On examination, he had left axillary and cervical lymphadenopathy.

\section{What is the most likely diagnosis?}
(a) Leptospirosis.
(b) Dengue fever.
(c) Cerebral malaria.
(d) Scrub typhus.
(e) Meningococcal meningitis.

5. A 23-year-old tour guide presented with fever that had lasted 8 days. She had been on a 2-week tour in South Africa, Israel and Greece and had returned 10 days previously. Examination revealed multiple skin lesions with a black scab and a widespread erythematous maculopapular rash. She was pregnant with gestational age approximately 10 weeks. 


\section{Which condition is she most likely to have?}
(a) Scrub typhus.
(b) Murine typhus.
(c) African tick bite fever.
(d) Indian tick typhus
(e) Mediterranean spotted fever.

6. A 40-year-old returning traveller presented to the travel health clinic with fever of 3 days' duration.
Which of the following features suggests melioidosis is a likely diagnosis?
(a) History of sea-bathing in the tropics.
(b) History of unprotected sexual intercourse with a sex worker while on holiday.
(c) Multiple lung abscesses.
(d) Petechial rash over the trunk.
(e) Thrombocytopenia.

7. A 30-year-old farmer presented with fever of 1 day's duration and profound shock. He had been exposed to flooding on the day prior to the onset of the illness. He was in shock (blood pressure $70 / 55 \mathrm{mmHg}$, pulse rate $110 \mathrm{bpm}$ ) and was in respiratory distress (respiratory rate 24 bpm, SaO2 88\% on room air). Examination revealed jaundice and bilateral lung crackles.

\section{Which of the following is the most appropriate treatment to commence immediately?}

(a) Ceftazidime and cotrimoxazole.

(b) Ceftazidime, vancomycin and metronidazole.

(c) Management for dengue haemorrhagic fever/dengue shock syndrome.

(d) Meropenem.

(e) Non-invasive ventilatory support.

8. A 25-year-old woman presented with fever that had lasted 3 days, abscesses in lung, liver and spleen, C-reactive protein $240 \mathrm{mg} / \mathrm{dL}$ (normal $<5$ ), and serum creatinine $300 \mu \mathrm{mol} / \mathrm{L}$ (normal $<90$ ). She was empirically commenced on meropenem. Subsequently the diagnosis was confirmed by blood culture as melioidosis. After 4 weeks of the intensive phase, she was febrile, CRP had decreased to $40 \mathrm{mg} / \mathrm{dL}$, creatinine had decreased to $110 \mu \mathrm{mol} / \mathrm{L}$, and the pulmonary, hepatic and splenic abscesses were found to have decreased in size. The clinician was considering switching to the eradication phase.

\section{Which feature best argues against switching to the eradication phase?}
(a) The blood culture was repeatedly positive in the first two weeks of the treatment.
(b) The CRP is still not within the normal range.
(c) The fever has still not settled.
(d) The liver and spleen abscesses are still not fully resolved.
(e) The raised serum creatinine has still not returned to normal.

9. A 35-year-old Southeast Asian woman on a vacation in the UK was admitted to the medical take with a 2-day history of fever and arthralgia. The diagnosis of dengue was confirmed. On day 4 of the illness, she developed abdominal pain and was found to have a rising haematocrit and plasma leakage into pleuro-peritoneal spaces in ultrasound examination. On the 36 th hour of critical phase monitoring, she was looking unwell and noted to have a tachycardia of $120 \mathrm{bpm}$ and blood pressure of $100 / 70 \mathrm{mmHg}$. The haematocrit dropped from $40 \%$ to $32 \%$.

\section{What is the likely complication?}
(a) Intravascular volume depletion due to leaking.
(b) Dengue myocarditis.
(c) Occult haemorrhage.
(d) Entering the recovery phase of DHF.
(e) Secondary bacterial infection leading to septic shock.

10. A 45-year-old male with serologically confirmed dengue fever, currently being managed as critical phase (CP) of dengue haemorrhagic fever, was found to have blood pressure of $110 / 90 \mathrm{mmHg}$ with pulse rate of $120 \mathrm{bpm}$. The haematocrit increased from $45 \%$ to $49 \%$. He was treated with two boluses $(10 \mathrm{ml} / \mathrm{kg})$ of normal saline. Subsequent haematocrit was $50 \%$ and tachycardia was persisting.
(a) Intravenous dextran 40 bolus $(10 \mathrm{ml} / \mathrm{kg})$.
(b) Intravenous crystalloid bolus.
(c) Blood transfusion.
(d) Inotrope infusion.
(e) Venesection.

What is the most appropriate immediate treatment?

\section{CME Respiratory medicine SAQ}

\section{Answers to the CME SAQ published in Clinical} Medicine in November 2021

$\begin{array}{llllllllll}\text { Q1 } & \text { Q2 } & \text { Q3 } & \text { Q4 } & \text { Q5 } & \text { Q6 } & \text { Q7 } & \text { Q8 } & \text { Q9 } & \text { Q10 } \\ \text { (d) } & \text { (d) } & \text { (a) } & \text { (b) } & \text { (e) } & \text { (b) } & \text { (c) } & \text { (e) } & \text { (d) } & \text { (b) }\end{array}$

